\title{
Progressive development of augmentation during long-term treatment with levodopa in restless legs syndrome: results of a prospective multi-center study
}

\author{
Birgit Högl · Diego García-Borreguero · Ralf Kohnen · Luigi Ferini-Strambi · \\ Georgios Hadjigeorgiou • Magdolna Hornyak · Al de Weerd · Svenja Happe • \\ Karin Stiasny-Kolster · Viola Gschliesser · Renata Egatz $\cdot$ Birgit Frauscher · \\ Heike Benes · Claudia Trenkwalder · Wayne A. Hening · Richard P. Allen
}

Received: 29 December 2008/Revised: 29 July 2009/Accepted: 14 August 2009/Published online: 11 September 2009 (C) Springer-Verlag 2009

\begin{abstract}
The European Restless Legs Syndrome (RLS) Study Group performed the first multi-center, long-term study systematically evaluating RLS augmentation under levodopa treatment. This prospective, open-label 6-month study was conducted in six European countries and included 65 patients (85\% treatment naive) with idiopathic RLS.
\end{abstract}

Wayne A. Hening: Deceased.

B. Högl $(\bowtie)$ · V. Gschliesser · B. Frauscher

Department of Neurology, Innsbruck Medical University,

Anichstr. 35, 6020 Innsbruck, Austria

e-mail: birgit.ho@i-med.ac.at

D. García-Borreguero · R. Egatz

Sleep Research Institute, Madrid, Spain

R. Kohnen

Research Pharmaceutical Services (RPS), Nuremberg, Germany

R. Kohnen

University of Erlangen-Nuremberg, Erlangen, Germany

L. Ferini-Strambi

Sleep Disorders Center,

University Vita-Salute San Raffaele, Milan, Italy

G. Hadjigeorgiou

Department of Neurology, Medical School,

University of Thessaly, Larissa, Greece

M. Hornyak

Department of Psychiatry and Interdisciplinary Pain Centre,

University of Freiburg, Freiburg, Germany

A. de Weerd

Sleep Center SEIN Zwolle, Zwolle, The Netherlands

S. Happe

Department of Clinical Neurophysiology,

Klinikum Bremen-Ost, Bremen, Germany
Levodopa was flexibly up-titrated to a maximum dose of $600 \mathrm{mg} /$ day. Presence of augmentation was diagnosed independently by two international experts using established criteria. In addition to the augmentation severity rating scale (ASRS), changes in RLS severity (International RLS severity rating scale (IRLS), clinical global

\author{
S. Happe \\ University of Goettingen, Goettingen, Germany \\ K. Stiasny-Kolster \\ Department of Neurology, Philipps University, \\ Marburg, Germany \\ H. Benes \\ Somnibene Institute for Medical Research and Sleep Medicine, \\ Schwerin, Germany \\ H. Benes \\ Neurology Department, \\ University of Rostock, Rostock, Germany \\ C. Trenkwalder \\ Paracelsus-Elena Hospital, Kassel, Germany \\ C. Trenkwalder \\ University of Goettingen, Goettingen, Germany \\ W. A. Hening \\ UMDNJ-RW Johnson Medical School, \\ New Brunswick, NJ, USA \\ R. P. Allen \\ Neurology and Sleep Medicine, \\ Johns Hopkins University, Baltimore, MD, USA
}


impression (CGI)) were analyzed. Sixty patients provided evaluable data, 35 completed the trial and 25 dropped out. Augmentation occurred in 60\% (36/60) of patients, causing $11.7 \%(7 / 60)$ to drop out. Median time to occurrence of augmentation was 71 days. The mean maximum dose of levodopa was $311 \mathrm{mg} /$ day (SD: 105). Patients with augmentation compared to those without were significantly more likely to be on higher doses of levodopa ( $\geq 300 \mathrm{mg}$, 83 vs. $54 \%, P=0.03$ ) and to show less improvement of symptom severity (IRLS, $P=0.039$ ). Augmentation was common with levodopa, but could be tolerated by most patients during this 6-month trial. Patients should be followed over longer periods to determine if dropout rates increase with time.

Keywords Restless legs syndrome (RLS) .

Augmentation - Diagnosis - Rating scale $\cdot$ Clinical study

\section{Introduction}

Levodopa was the first dopaminergic therapy investigated for the treatment of restless legs syndrome (RLS) [1, 25] and is licensed for the treatment of RLS in certain European countries. Evidence for the efficacy of levodopa was demonstrated in the first placebo-controlled short-term clinical trials of dopaminergic treatment in RLS [6, 8, 9, $14,20]$. However, more than a decade ago augmentation of RLS symptoms was noted as a serious complication of levodopa therapy [2]. Augmentation is a worsening of RLS symptom severity characterized by the occurrence of RLS symptoms earlier in the day, by a shorter latency to symptoms at rest, increased intensity of symptoms, and a spreading of RLS symptoms to previously unaffected areas of the body [3] compared to the status at start of treatment or to favourable initial response. Trenkwalder et al. [21] reported that $35 \%$ ( 8 of 23 ) of all treated patients during a 1 year open-label study of levodopa/benserazide therapy discontinued the trial prematurely due to a time-shift towards intolerable symptoms during the day. In a recent double-blind, long-term (6 months) trial comparing the dopamine agonist cabergoline to levodopa/benserazide [23], $9.8 \%$ of patients treated with levodopa developed augmentation requiring premature discontinuation. In summary, the results of previous studies suggest a substantial risk for augmentation with levodopa therapy. There has not, however, been any study specifically designed to prospectively evaluate the development of augmentation with levodopa treatment systematically by NIH criteria for augmentation and with the augmentation severity rating scale (ASRS).

In the year 2003, the European RLS Study Group (EURLSSG) decided to conduct an open-label study with levodopa to validate an ASRS and to evaluate prospectively and systematically the incidence and clinical characteristics of augmentation [11]. In this manuscript, we report the clinical outcome of this first study designed to evaluate RLS augmentation with levodopa treatment.

\section{Methods}

Design

This was a 6-month multi-center, open-label trial with a flexible dose of levodopa. The levodopa dose was adjusted according to clinical needs and adverse effects. The investigators determined the optimal levodopa dose by means of weekly contacts during the first month (weeks 1 and 3 by phone, and site visits on weeks 2 and 4). Patients then entered the maintenance period of the study for a further 5 months and were monitored with monthly site visits. During the initial dose adjustment period, according to the protocol, levodopa/benserazide had to be up-titrated from 100/25 mg per day to a minimum dose of 200/50 mg per day, but could be further increased to a maximum dose of $600 / 150 \mathrm{mg}$ per day, although this maximum dose was never reached during the study. Dosage titration and adjustment were guided by two principles: (a) the levodopa dose was to be increased until elimination of all clinically meaningful symptoms, (b) once a sufficient dosage was achieved, that dosage should be kept as stable as possible over time. Dose adjustments were permitted throughout the trial.

\section{Patients}

The study was designed to include patients who had never before been treated with dopaminergic drugs (levodopa, dopamine agonists), who were aged between 18 and 80 years and who met the diagnostic criteria for RLS as established by the International RLS Study Group [3]. All patients were judged by the investigators as severe enough to require therapy for their RLS. Patients were excluded from the study if RLS symptoms at baseline occurred before 6 p.m. Further exclusion criteria included other severe primary sleep disorders, neurological, psychiatric, and pain disorders or severe medical and surgical conditions, as well as clinically relevant laboratory abnormalities. Concomitant therapy with any other psychotropic medication which could have an influence on RLS symptoms (e.g., opioids, antiepileptics) was not permitted with the exception of treatment with hypnotics, anxiolytic drugs, antidepressants, benzodiazepines, and other therapies if the treatment had been started at least 4 weeks prior to enrollment into the study and the dose could be kept stable throughout the trial. Domperidone could be administered in the event of gastrointestinal complaints such as nausea. 


\section{Outcome measures}

Severity of RLS symptoms was evaluated at all visits with the International RLS severity scale (IRLS) [26], the RLS6 scales [15], and the clinical global impressions (CGI) [16]. Severity of augmentation was assessed with the ASRS [11]. The ASRS evaluates the severity of augmentation by comparing the status before initiation of RLS therapy to assessments during therapy. The ASRS is based on an earlier onset of symptoms, prolonged latency to symptoms at rest and spreading of symptoms to other body parts. The severity of augmentation was graded from 0 to 24 (total score).

Other outcome measures were treatment satisfaction as measured with the treatment satisfaction questionnaire for medication (TSQM) [4], and quality of life (RLS quality of life instrument (RLS-QLI) [5]). All rating scales were assessed at baseline and then in monthly intervals or, in case of dropouts, at the final visit.

"Gold standard" diagnosis of augmentation was based on an expert rating. To obtain these expert ratings, data of all patients were submitted to two highly experienced experts in augmentation (Diego Garcia-Borreguero and Richard P. Allen) asking for an independent assessment on the presence (AUG) or absence of augmentation (N-AUG) as based on published diagnostic criteria [3]. Experts discussed and resolved any divergent ratings so that the final diagnosis was a consensus diagnosis of both. Data provided to the experts comprised the ASRS items, the IRLS and the RLS-6 scores, the CGI ratings, and dosage of levodopa from all visits of the study.

\section{Statistical analyses}

Efficacy was analyzed in an exploratory manner for all patients who had at least one post-baseline IRLS total score (modified intention to treat set). Efficacy analysis was based on comparisons of changes between the baseline visit and the patients' individual final visit using the IRLS total score, the RLS-6 scales and the CGI. If patients withdrew prematurely from the study, the last observation was carried forward.

Patients were stratified according to occurrence of augmentation as diagnosed by the independent experts. Comparisons between AUG and N-AUG were performed with two-sample tests for quantitative (Mann-Whitney $U$ test) or qualitative (Fisher's exact test) variables. Time to event data was described with a Kaplan-Meier statistic.

\section{Sample size}

The number of patients to be included in this trial was based upon the requirements for assessing the validity of the ASRS [11]. It was expected from earlier studies [2, 21] that approximately half of all patients would experience augmentation, thus 60 patients were planned to be included in this trial.

\section{Results}

\section{Centers and patients}

Approval from the local ethics committees was achieved in all of the eight participating centers in six European countries. Between October 2003 and February 2005, 65 patients were enrolled into the trial. Two patients withdrew their written consent to participate prior to any intake of levodopa. Three other patients were withdrawn early after baseline by the local investigators due to deviations from the study protocol [no dose increase to $200 \mathrm{mg}$ levodopa possible (1 patient), previous treatment with dopamine agonists (2 patients)]. Sixty RLS patients were finally treated and evaluated. Of those, 25 patients $(41.7 \%)$

Table 1 Demographic and clinical characteristics of study patients

\begin{tabular}{lllr}
\hline Statistics & $\begin{array}{l}\text { All patients } \\
(N=60)\end{array}$ & $\begin{array}{l}\text { With augmentation } \\
(N=36)\end{array}$ & $\begin{array}{l}\text { No augmentation } \\
(N=24)\end{array}$ \\
\hline Age in years, $\mathrm{M} \pm \mathrm{SD}$ & $52.6 \pm 12.8$ & $51.5 \pm 12.0$ & $53.3 \pm 13.4$ \\
Gender & & & 0.5373 \\
$\quad$ Male, $N(\%)$ & $22(36.7)$ & $10(27.8)$ & $12(50.0)$ \\
Female, $N(\%)$ & $38(63.3)$ & $26(72.2)$ & $12(50.0)$ \\
Body Mass Index $\left(\mathrm{kg} / \mathrm{m}^{2}\right), \mathrm{M}(\mathrm{SD})$ & $25.4(4.1)$ & $25.1(4.2)$ & $25.8(3.8)$ \\
Diagnosis confirmed by PSG, $N(\%)$ & $25(41.7)$ & $14(38.9)$ & $11(45.8)$ \\
Familial history of RLS, $N(\%)$ & $25(41.7)$ & $18(50.0)$ & $7(29.2)$ \\
Ferritin at baseline $(\mu \mathrm{g} / \mathrm{L}), \mathrm{M} \pm \mathrm{SD}(\mathrm{Md})$ & $101 \pm 70(87)$ & $82 \pm 47(77)$ & $131 \pm 88(126)$ \\
IRLS total score at baseline, $(\mathrm{M} \pm \mathrm{SD})$ & $24.7 \pm 5.2$ & $24.0 \pm 4.0$ & $25.7 \pm 6.5$
\end{tabular}

$P$ value associated with 2-sample tests (Mann-Whitney $U$ test, Fisher's exact test) to compare patients with or without augmentation $M$ arithmetic mean, $S D$ standard deviation, $N$ number of patients, $M d$ Median, $P S G$ polysomnography 
discontinued the study prematurely. The most frequent individual reasons for dropout were lack of efficacy $(n=7$ patients, $11.7 \%$ of all patients) and augmentation $(n=7$, $11.7 \%$ ). The other 11 patients discontinued due to adverse events $(n=3,5.0 \%)$, withdrawal of consent $(n=2,3.3 \%)$ or loss to follow-up $(n=6,10 \%)$. Demographic and clinical characteristics of the intention to treat population $(n=60)$ are reported in Table 1, both for the total population and stratified by occurrence of augmentation according to the experts [yes (AUG): $n=36$, no (N-AUG): $n=24$, see below]. Contrary to the study protocol, six patients in two centers received prior dopaminergic therapy ( $n=3$ levodopa, $n=4$ dopamine agonists). Augmentation occurred in three of these patients (in 1 patient with levodopa pre-treatment).

AUG and N-AUG differed in several of the baseline characteristics: augmenters were more frequently females and/or had a positive family history of RLS. They also had a slightly lower serum ferritin measure at baseline, although none of these differences reached significance. Augmenters had more frequently experienced lack of efficacy of at least one previous treatment therapy. Previously treated and non-treated subgroups did not differ in their levodopa dosage or in the response to levodopa.
Treatment

On average, the patients were treated for approximately 4.5 months of the planned treatment duration of 6 months with a mean levodopa dose of $311 \mathrm{mg} /$ day (Table 2). There was no difference in the average levodopa dose between AUG and N-AUG $(P=0.2886$, Mann-Whitney $U$ Test $)$. There was a trend towards higher levodopa dose per $\mathrm{kg}$ body weight in AUG that just missed our significance criteria $(P=0.0697, U$ test $)$. In addition, the number of patients who were treated with at least $300 \mathrm{mg}$ levodopa per day was higher in AUG (83.3\%) than in N-AUG (54.2\%) $(P=0.0312$, Fisher's exact test, 2-sided).

\section{Augmentation}

After inspection of the original data of each patient, the two independent experts classified 36 patients $(60 \%$ of all patients) to have definitely experienced augmentation during levodopa treatment. Both groups differed in the ASRS total score $(P<0.0001)$ : the mean and standard deviations of the worst (maximum) total score (3 items, range 0 to 24) were $7.4 \pm 4.0$ for AUG versus $2.0 \pm 2.7$ in N-AUG. Regarding the range of the ASRS total score, the mean ASRS total score indicates that in AUG the severity

Table 2 Levodopa treatment stratified by expert rating on augmentation

\begin{tabular}{|c|c|c|c|c|}
\hline Statistics & $\begin{array}{l}\text { All patients } \\
(N=60)\end{array}$ & $\begin{array}{l}\text { With augmentation } \\
(N=36)\end{array}$ & $\begin{array}{l}\text { No augmentation } \\
(N=24)\end{array}$ & $P$ values \\
\hline \multicolumn{5}{|c|}{ Duration of treatment (days) } \\
\hline $\mathrm{M} \pm \mathrm{SD}$ & $140 \pm 62$ & $138 \pm 60$ & $143 \pm 65$ & \multirow[t]{2}{*}{0.7632} \\
\hline Range & $13-202$ & $17-202$ & 13-202 & \\
\hline \multicolumn{5}{|c|}{ Maximum LD dose (mg) } \\
\hline $\mathrm{M} \pm \mathrm{SD}$ & $311 \pm 105$ & $324 \pm 98$ & $292 \pm 114$ & \multirow[t]{2}{*}{0.2886} \\
\hline Range & $50-500$ & $50-500$ & $100-500$ & \\
\hline \multicolumn{5}{|c|}{ Maximum LD dose per $\mathrm{kg}$ body weight $(\mathrm{mg} / \mathrm{kg})$} \\
\hline $\mathrm{M} \pm \mathrm{SD}$ & $4.7 \pm 1.7$ & $5.0 \pm 1.6$ & $4.2 \pm 1.8$ & \multirow[t]{2}{*}{0.0697} \\
\hline Range & $1.39-8.40$ & $2.35-8.40$ & $1.39-8.00$ & \\
\hline \multicolumn{5}{|c|}{ Maximum LD dose (mg), $N(\%)$} \\
\hline 50 & $1(1.7)^{\mathrm{a}}$ & $1(2.8)^{\mathrm{a}}$ & 0 & \\
\hline 100 & $2(3.3)$ & 0 & $2(8.3)$ & \\
\hline 200 & $14(23.2)$ & $5(13.9)$ & $9(37.5)$ & \\
\hline 300 & $21(35.0)$ & $18(50.0)$ & $3(12.5)$ & \\
\hline 400 & $17(28.3)$ & $8(22.2)$ & $9(37.5)$ & \\
\hline 500 & $5(8.3)$ & $4(11.1)$ & $1(4.2)$ & \\
\hline$>200$ & $43(71.7)$ & $30(83.3)$ & $13(54.2)$ & 0.0312 \\
\hline
\end{tabular}

$P$ value associated with 2-sample tests (Mann-Whitney $U$ test, Fisher's exact test) to compare patients with or without augmentation

$M$ arithmetic mean, $S D$ standard deviation, Range minimum-maximum, $L D$ levodopa

a One patient who did not tolerate $100 \mathrm{mg}$ L-Dopa, early discontinuation 


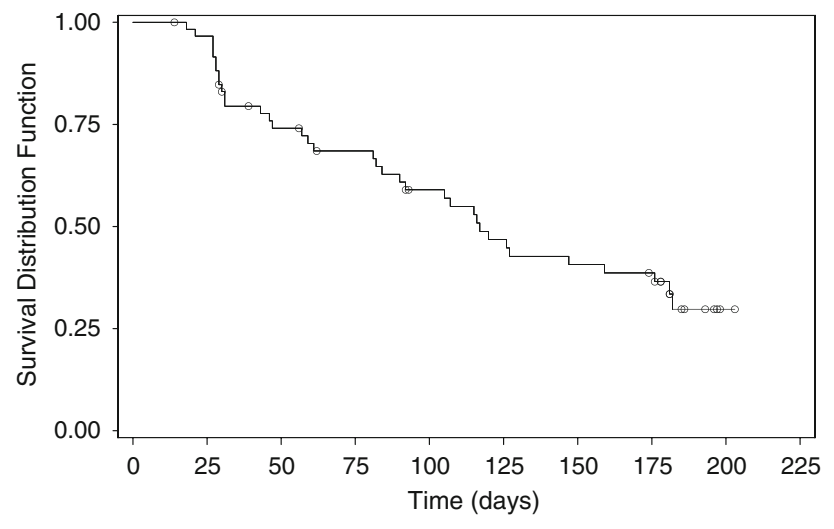

Fig. 1 This figure shows a Kaplan-Meier survival curve on the occurrence of augmentation according to expert rating throughout the progress of the trial (treatment days on $X$-axis). The first assessment of the ASRS was performed on day 29. Patients with no augmentation are censored with the date of their last observation within the study (circles)

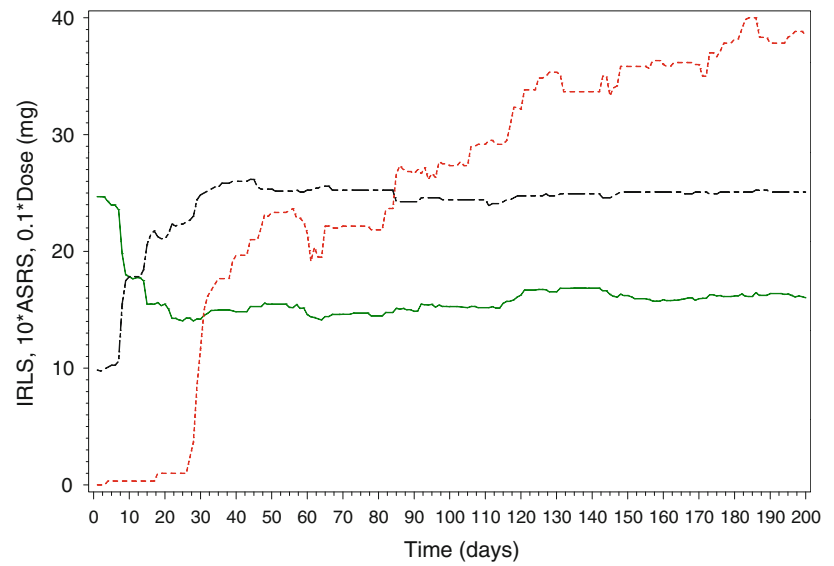

Fig. 2 IRLS (green line): original values, levodopa dose (black line) $\times 0.1$. ASRS total score (red line) $\times 10$ to achieve comparable scale level for all three variables. Values were carried forward for all patients until a change in either variable occurred. In dropouts, the last observed value was carried forward for all three variables

of augmentation was, on average, in the lower range of the scale.

In Fig. 1, time to occurrence of augmentation is presented as a Kaplan-Meier plot from a life-table analysis. The date of onset of augmentation was defined by the independent experts. Median time to augmentation was 71 days with a range between 18 and 182 days. Figure 1 illustrates that augmentation can occur at any time within 6 months of start of therapy. Figure 2 illustrates the change over time of augmentation severity, levodopa dose, and RLS severity according to the IRLS total score during the course of the study. In this figure, we carried forward available data for days between visits until a measure changed or until the end of the study in patients who discontinued the trial prematurely (LOCF). While the total scores on the augmentation-specific rating scale ASRS increased progressively over time, the IRLS total score and the levodopa dose remained stable during maintenance treatment, indicating that treatment efficacy was maintained on average. The figure suggests that the process of augmentation contains specific features that are not reflected in severity scales, and that it might be different from pharmacological tolerance.

\section{Clinical outcome}

Table 3 summarizes baseline-endpoint comparisons for the scale measures of severity of RLS, quality of life, and satisfaction with treatment based on LOCF. In general, improvements (as based on IRLS or CGI) were larger in N-AUG than in AUG. In the RLS-6 scales, however, only severity at bedtime was significantly more improved in N-AUG $(P=0.018)$, but quality of sleep and daytime tiredness showed similar changes across groups. In the TSQM, effectiveness of and global satisfaction with levodopa therapy were rated more favorably in N-AUG than in AUG.

Safety

Safety monitoring was performed at each visit with a focus on established side effects of levodopa/benserazide requiring intervention. Three patients discontinued the study prematurely due to adverse events, two augmenters (subjectively reported impaired cognitive ability in one patient, impaired coordination and emotional disturbance in the other), and one patient without augmentation due to tiredness during the day, nausea and nightmares.

\section{Discussion}

This prospective, open-label, multi-center study confirms the high risk for augmentation during levodopa therapy of RLS patients. Augmentation was diagnosed in $60 \%$ of all analyzable patients and occurred at all doses of levodopa between 50 and $500 \mathrm{mg} /$ day. Furthermore, augmentation could occur at any time during the 6 month treatment period and its prevalence increased progressively with time (Fig. 1). In addition, its severity also increased with the duration of levodopa therapy (Fig. 2).

A relationship between the incidence of augmentation and higher levodopa dosages is supported by higher rates of augmenters than non-augmenters who were treated with $300 \mathrm{mg}$ per day or higher when augmentation occurred. In addition, we found a marginally positive correlation between levodopa dose and augmentation when the 
Table 3 Comparisons of patients with or without augmentation in clinical outcome scores (change from baseline to individual last visit during treatment phase)

\begin{tabular}{|c|c|c|c|c|c|}
\hline \multirow[t]{2}{*}{ Scale } & \multirow[t]{2}{*}{ Subscale } & \multirow{2}{*}{$\begin{array}{l}\text { Baseline } \\
\text { All patients }\end{array}$} & \multicolumn{2}{|c|}{ Change from baseline } & \multirow{2}{*}{$P$ value } \\
\hline & & & With augmentation & No augmentation & \\
\hline IRLS & Total score & $24.7 \pm 5.2$ & $-6.4 \pm 11.2$ & $-12.4 \pm 10.7$ & 0.039 \\
\hline \multirow[t]{4}{*}{ CGI } & 1. Severity & $4.5 \pm 0.8$ & $-0.7 \pm 1.8$ & $-1.9 \pm 1.6$ & 0.036 \\
\hline & 2. Change in condition ${ }^{\mathrm{a}}$ & n.a. & $3.0 \pm 1.9$ & $1.8 \pm 1.2$ & 0.004 \\
\hline & 3. Therapeutic effect ${ }^{\mathrm{a}}$ & n.a. & $2.4 \pm 1.3$ & $1.5 \pm 0.9$ & 0.005 \\
\hline & 4. Side effects ${ }^{\mathrm{a}}$ & n.a. & $1.7 \pm 0.9$ & $1.4 \pm 0.6$ & 0.265 \\
\hline \multirow[t]{6}{*}{ RLS-6 } & Severity bedtime & $5.7 \pm 3.0$ & $-2.3 \pm 4.2$ & $-4.8 \pm 3.0$ & 0.018 \\
\hline & Severity at night & $5.1 \pm 2.9$ & $-1.7 \pm 3.8$ & $-3.4 \pm 3.3$ & 0.101 \\
\hline & Severity day at rest & $4.0 \pm 2.9$ & $0.3 \pm 3.8$ & $-1.6 \pm 3.1$ & 0.060 \\
\hline & Severity day active & $1.2 \pm 1.7$ & $-0.2 \pm 2.0$ & $-0.6 \pm 1.6$ & 0.301 \\
\hline & Sleep quality & $6.7 \pm 1.9$ & $-2.3 \pm 3.7$ & $-3.0 \pm 3.1$ & 0.346 \\
\hline & Daytime tiredness & $4.7 \pm 3.1$ & $-0.9 \pm 3.9$ & $-2.3 \pm 3.6$ & 0.142 \\
\hline \multirow[t]{4}{*}{ QLI } & Social function & $66.5 \pm 24.5$ & $+8.6 \pm 24.7$ & $+22.2 \pm 25.7$ & 0.053 \\
\hline & Daily function & $71.3 \pm 12.8$ & $+6.8 \pm 19.9$ & $+5.6 \pm 24.5$ & 0.984 \\
\hline & Sleep quality & $34.8 \pm 18.2$ & $+15.3 \pm 27.2$ & $+27.3 \pm 28.4$ & 0.189 \\
\hline & Emotional wellbeing & $57.1 \pm 30.9$ & $+10.0 \pm 37.5$ & $+23.2 \pm 34.0$ & 0.168 \\
\hline \multirow[t]{4}{*}{ TSQM } & Effectiveness $^{\mathrm{a}}$ & n.a. & $57.6 \pm 27.5$ & $71.5 \pm 31.4$ & 0.049 \\
\hline & Side effects ${ }^{\mathrm{a}}$ & n.a. & $56.3 \pm 29.2$ & $60.6 \pm 32.6$ & 0.463 \\
\hline & Convenience $^{\mathrm{a}}$ & n.a. & $79.6 \pm 16.7$ & $86.5 \pm 16.4$ & 0.076 \\
\hline & Global satisfaction $^{\mathrm{a}}$ & n.a. & $50.6 \pm 32.9$ & $75.8 \pm 30.1$ & 0.003 \\
\hline
\end{tabular}

IRLS, CGI item 1, RLS-6: negative signs indicate improvement; CGI 2 to 4: score 1 = best condition; $Q L I$ positive signs indicate improvement; TSQM $100=$ best condition

$\$ P$ values associated with the Mann-Whitney $U$ test

${ }^{a}$ No baseline values are available for these scales, they represent ratings of change

absolute dose was related to the patients' body weight. However, we cannot make a valid statement on the robustness of this relationship due to a large heterogeneity of treatment strategies in the participating centres. Although $70 \%$ of the patients treated with doses higher than $200 \mathrm{mg}$ developed augmentation, the remaining $30 \%$ included some who tolerated even higher doses without any indication that increased doses above $300 \mathrm{mg}$ further increased the risk of augmentation. We do not know whether those with no augmentation despite high doses of levodopa would also have become augmenters with additional time on the same doses.

Within the 6-month treatment period, 7 out of 36 patients $(19.4 \%)$ with augmentation or $11.7 \%$ of the total population had to discontinue treatment due to augmentation. This finding is similar to a discontinuation rate of $9.8 \%$ under levodopa/benserazide in the comparison trial with cabergoline [23] over 6 months and significantly higher than under cabergoline $(4.0 \%)$. The overall results of the ASRS in our trial show that severity of augmentation was in the lower range of the scale in the majority of all affected patients. This finding reflects the outcome of our study, unreported until now, that 29/36 (80.6\%) of patients with augmentation have tolerated this worsening of their disorder, e.g., if symptoms occur earlier than usual or during the day at rest. This finding underscores the point that augmentation has varying degrees of severity, and in many cases might be tolerable or compensated for. Therefore, a criterion to distinguish tolerable from clinically relevant augmentation is necessary as defined in the new guidelines on augmentation [12]. First clinical recommendations on how to treat the varying degrees of augmentation have been proposed [13]. Further studies should address whether the severity of augmentation increases or varies over time, as it does not seem to be a stationary process but in general appears to become more common with longer duration of treatment.

This study also shows that patients who develop augmentation have a less favorable treatment outcome compared to those who do not develop augmentation. Clinically relevant response to levodopa treatment was more pronounced in patients without augmentation than in augmenters, as shown by larger improvements of the IRLS total score, the RLS-6 scales, the CGI and the patients' assessment of the treatment's effectiveness and their global satisfaction with the levodopa therapy. 
In the literature, several sometimes contradictory risk factors for augmentation have been suggested to date: type of drug (higher under levodopa than under dopamine agonists), higher doses of drugs, duration of treatment, previous augmentation or tolerance, severity of RLS at start of therapy, familial RLS, secondary RLS and lack of neuropathy [18, 22, 24]. However, such associations are either controversial or controlled data are lacking [12]. The sample size in our study was too small for multivariate regression analyses; however, longer duration of treatment, higher dose, longer duration of symptoms, and any previous RLS treatment were found more frequently in AUGs than in N-AUGs treated with levodopa. In addition, low serum ferritin level has been found in two independent previous studies to be a risk factor for augmentation $[10,24]$ and the association was marginally significant in our study. Considering that some patients treated with high dosages of levodopa (>300 mg) for 6 months did not experience augmentation, future research might identify protective factors for augmentation, e.g., a genetic disposition.

Besides the description of a progressive development of augmentation associated with duration of treatment during long-term levodopa therapy, a main contribution of this trial to augmentation research is the identification of tolerable and intolerable severity of this treatment complication: augmentation with ASRS total severity scores in the lower range of the scale might be tolerated by many affected patients assuming it does not become progressively worse with longer treatment duration. Similar experiences have been reported from trials with dopamine agonists [7, 17, 19]. The recent revision of the criteria for augmentation [12] proposed conditions for clinically relevant augmentation, such as the need for the patients to change therapy or to adjust their daily activities. In light of this new concept of augmentation, the clinical relevance of most previous reports on augmentation might need to be re-assessed since they were based on a dichotomized concept of augmentation (presence or absence) and used a variety of different definitions and criteria. Augmentation rates as reported in the literature for dopamine agonists were: pergolide 15-27\%; pramipexole 8-56\%, cabergoline 3-9\%, compared to levodopa 10-72\% (for an overview see Garcia-Borreguero et al. [13]). In particular, the dosage level of levodopa has to be controlled in such re-analyses of previous data. It is also important to realize that we have hardly any data on very long durations of treatment (e.g., 10 years), so at this point we do not know the extent to which augmentation may or may not gradually worsen with duration of treatment. Obtaining these data should be critical for considering future treatment options, including whether or not early detection of mild augmentation has any clinical relevance for preventing development of more serious clinically significant augmentation.

From a clinical point of view, our study suggests that any clinician who prescribes levodopa to RLS patients should be aware of the potential occurrence of augmentation at any time and any dose during treatment. In accordance with recommendations on prevention of augmentation [13], levodopa should be applied to RLS treatment at maximum dosages of $200 \mathrm{mg} /$ day or $3 \mathrm{mg} / \mathrm{kg}$ body weight. We suggest that a careful ongoing review of patients on levodopa therapy is warranted and that patient education should include an explanation about the possibility and nature of augmentation.

This study was designed in 2003 as one of the first prospective multicenter trials on drug-induced augmentation (see also Trenkwalder et al. [23]). The intention of the authors was to administer levodopa close to routine practice. Therefore, the selection criteria for study patients were broad and protocol deviations such as previous therapy with dopaminergic drugs were tolerated. In 2004, what is nowadays common knowledge in RLS treatment was still unknown, such as the increased risk of augmentation under high dosages of levodopa or the influence of concomitant medication such as antidepressants on onset or aggravation of RLS symptoms. On the other hand, our current knowledge on levodopa-induced augmentation was supported substantially by the results of this trial as indicated by the recommendation to limit the maximum dosage of levodopa to a very low level of $200 \mathrm{mg} /$ day. We also introduced the concept of diagnosis of augmentation by use of an expert board instead of individual investigators to achieve as much standardized evaluation of the study data as possible. This approach is currently state of the art in augmentation research.

Acknowledgment This study was supported by an unrestricted educational grant from Pharmacia (now Pfizer) USA to Diego García-Borreguero which was used for compensation of travel expenses for a planning meeting. Development of the study protocol and the case record forms, study management, statistical analysis and reporting was provided on a non-profit basis by RPS Germany (Ralf Kohnen).

\section{References}

1. Akpinar S (1982) Treatment of restless legs syndrome with levodopa plus benserazide. Arch Neurol 39:739

2. Allen RP, Earley CJ (1996) Augmentation of the restless legs syndrome with carbidopa/levodopa. Sleep 19:205-213

3. Allen RP, Picchietti D, Hening WA et al (2003) Restless legs syndrome: diagnostic criteria, special considerations, and epidemiology. A report from the restless legs syndrome diagnosis and epidemiology workshop at the National Institutes of Health. Sleep Med 4:101-119 
4. Atkinson MJ, Sinha A, Hass SL et al (2004) Validation of a general measure of treatment satisfaction, the treatment satisfaction questionnaire for medication (TSQM), using a national panel study of chronic disease. Health Qual Life Outcomes 2:12

5. Atkinson MJ, Allen RP, DuChane J et al (2004) Validation of the restless legs syndrome quality of life instrument (RLS-QLI): findings of a consortium of national experts and the RLS Foundation. Qual Life Res 13:679-693

6. Benes H, Kurella B, Kummer J, Kazenwadel J, Selzer R, Kohnen $R$ (1999) Rapid onset of action of levodopa in restless legs syndrome: a double-blind, randomized, multicenter, crossover trial. Sleep 22:1073-1081

7. Benes H, Heinrich CR, Ueberall MA, Kohnen R (2004) Longterm safety and efficacy of cabergoline for the treatment of idiopathic restless legs syndrome: results from an open-label 6-month clinical trial. Sleep 27:674-682

8. Brodeur C, Montplaisir J, Godbout R, Marinier R (1988) Treatment of restless legs syndrome and periodic movements during sleep with L-dopa: a double-blind, controlled study. Neurology 38:1845-1848

9. Collado-Seidel V, Kazenwadel J, Wetter TC et al (1999) A controlled study of additional sr-L-dopa in L-dopa-responsive restless legs syndrome with late-night symptoms. Neurology $52: 285-290$

10. Frauscher B, Gschliesser V, Brandauer E et al (2009) The severity range of restless legs syndrome (RLS) and augmentation in a prospective patient cohort: association with ferritin levels. Sleep Med 10:611-615

11. Garcia-Borreguero D, Kohnen R, Högl B et al (2007) Validation of the augmentation severity rating scale (ASRS): a multicentric, prospective study with levodopa on restless legs syndrome. Sleep Med 8:455-463

12. Garcia-Borreguero D, Allen RP, Kohnen R et al (2007) Diagnostic standards for dopaminergic augmentation of restless legs syndrome: report from a World Association of Sleep MedicineInternational Restless Legs Syndrome Study Group consensus conference at the Max Planck Institute. Sleep Med 8:520-530

13. Garcia-Borreguero D, Allen RP, Benes H et al (2007) Augmentation as a treatment complication of restless legs syndrome: concept and management. Mov Disord 22(Suppl 18):S476-S484
14. Kaplan PW, Allen RP, Buchholz DW, Walters JK (1993) A double-blind, placebo-controlled study of the treatment of periodic limb movements in sleep using carbidopa/levodopa and propoxyphene. Sleep 16:717-723

15. Kohnen R, Oertel WH, Stiasny-Kolster K, Benes H, Trenkwalder C (2004) Severity rating of restless legs syndrome: Validation of the RLS-6 scales. Sleep 27 (Abstract Suppl):A304

16. National Institute of Mental Health (NIMH) (1976) Early clinical drug evaluation unit (ECDEU). Clinical global impressions. In: Guy W (ed) ECDEU assessment manual for psychopharmacology, Revised edn. NIMH, Rockville, pp 218-222

17. Ondo W, Romanyshyn J, Vuong KD, Lai D (2004) Long-term treatment of restless legs syndrome with dopamine agonists. Arch Neurol 61:1393-1397

18. Ondo WG (2005) Restless legs syndrome. Curr Neurol Neurosci Rep 5:266-274

19. Stiasny-Kolster K, Benes H, Peglau I et al (2004) Effective cabergoline treatment in idiopathic restless legs syndrome. Neurology 63:2272-2279

20. Trenkwalder C, Stiasny K, Pollmächer T et al (1995) L-dopa therapy of uremic and idiopathic restless legs syndrome: a double-blind, crossover trial. Sleep 18:681-688

21. Trenkwalder C, Collado Seidel V, Kazenwadel J et al (2003) One-year treatment with standard and sustained-release levodopa: appropriate long-term treatment of restless legs syndrome? Mov Disord 18:1184-1189

22. Trenkwalder C, Paulus W, Walters AS (2005) The restless legs syndrome. Lancet Neurol 4:465-475

23. Trenkwalder C, Benes H, Grote L et al (2007) Cabergoline compared to levodopa in the treatment of patients with severe restless legs syndrome: results from a multi-center, randomized, active controlled trial. Mov Disord 22:696-703

24. Trenkwalder C, Högl B, Benes H, Kohnen R (2008) Augmentation in restless legs syndrome is associated with low ferritin. Sleep Med 9:572-574

25. von Scheele C (1986) Levodopa in restless legs. Lancet 2:426427

26. Walters AS, LeBrocq C, Dhar A et al (2003) Validation of the International Restless Legs Syndrome Study Group rating scale for restless legs syndrome. Sleep Med 4:121-132 